\title{
Analysis of the Clinicopathological Features of Hepatocellular Carcinoma in Elderly Patients
}

\author{
Jing-Lin X, ${ }^{1}$ Sharma D, ${ }^{1}$ Bing-Hui $Y,{ }^{1}$ Bo-heng Z, ${ }^{1}$ Zeng-Chen $M,{ }^{1}$ Zhi-Quan W, Jia F, ${ }^{1}$ Xin Da Z, \\ Lun- Xiu $Q,{ }^{\prime}$ Zhao-You $T^{1}$ \\ 'Liver Cancer Institute, Zhongshan Hospital, Fudan University, Shanghai 200032, P.R., China
}

\begin{abstract}
The peak age of onset of hepatocellular carcinoma (HCC) is continually increasing worlwide. This study aims to evaluate whether there exists any significant difference in the clinicopathological features between younger- and elderly-HCC.1082 Consecutive patients with HCC who underwent liver resection at Liver Cancer Institute, Zhongshan Hospital, Fudan University from 1995 to 1998 were studied. The patients were divided into elderly-HCC ( $\geq 65$ years of age) and younger-HCC $(<65$ years of age). Important clinicopathological features of the patients and postoperative survival rates were compared between these two groups. Among 1082 patients studied, 108 were elderly-HCC and 974 were younger-HCC. The resection rate of the elderly-HCC was significantly higher than that of the younger-HCC. The 1, 3 and 5-year survival rates of the elderly-HCC were not significantly different from those of the younger-HCC. Compared with the younger-HCC, the elderly-HCC had (1) less HBsAg-positive rate; (2) more frequent anti-HCV positivity ; (3) lower proportion with AFP value $\geq 400 \mu \mathrm{g} / \mathrm{dl}$; (4) a relatively small tumor diameter; (5) higher proportion of stage I-II patients; (6) a relatively low metastasis rate. However, there were no statistically significant differences in other clinicopathological features (including gender, symptoms, tumor number, tumor venous invasion, tumor differentiation, capsular formation, type of cirrhosis) between the two groups. There is a certain extent difference in clinicopathological features between elderly and younger-HCC patients, but the postoperative survival rate is comparable between the two groups.
\end{abstract}

Key words: age, clinicopathological features, hepatocellular carcinoma, prognosis

\section{INTRODUCTION}

Hepatocellular carcinoma (HCC) is one of the most common malignancies in China, which accounts for $45 \%$ of total HCC deaths in the world annually. The peak age of onset of $\mathrm{HCC}$ is continually increasing. Yang $\mathrm{BH}$ et al. analyzed the clinical data of 3250 patients with primary liver cancer, and compared with the datas of 30 years ago, found that the mean age of onset of HCC increased from 43.7 to $49.1 .^{1}$ The peak age of onset of HCC varies with the geographical location as fifty to sixty years of age in Taiwan and older than sixty years of age in the United States and western Europe, while older than seventy years of age in Japan..$^{2-4}$

In clinical practice, more and more surgical resection is performed in elderly HCC patients, particularly in China and Japan. Clinicopathological features and prognosis in elderly HCC patients compared to HCC patients in relatively younger group needs to be further explored for more appropriate approach for the management. There are reports from Japan indicating that younger HCC patients had higher rates of hepatitis B surface antigen (HBsAg) carrier, higher serum alpha-fetoprotein
Correspondence:

Xia Jing-Lin

Liver Cancer Institute, Zhongshan Hospital

Fudan University, 200032, Shanghai, China

Email: xiajinglin@hotmail.com 
Jing-Lin et al. Analysis of the Clinicopathological Features of Hepatocellular Carcinoma in Elderly Patients

(AFP) level and better liver functions compared with those of elderly patients. ${ }^{5}$ In spite of having largest number of HCC patients in China, there are only few reports about these issues. In this study, we evaluated the clinicopathological features of 1082 patients who underwent surgical resection of HCC from 1995 to 1998. Patients were divided into 2 groups according to their age and we tried to explore the characteristics of elderly HCC patients in terms of clinicopathological features and postoperative prognosis.

\section{MATERIAL AND METHODS}

Consecutive patients who had undergone surgical resection (including those who received open-close surgery) for the treatment of HCC at Zhongshan Hospital, Fudan University from January 1995 to December 1998 were enrolled in our study. Diagnoses of HCC were made by clinical examination, laboratory parameters, radiological investigations and finally confirmed by histopathological examination of the surgical specimen. Patients were followed up to December 2001.

Patients below 65 years of age were defined as the younger group while patients of 65 years of age or above were defined as the elderly group. The following clinicopathological features before and after surgical resection were included namely gender, symptoms, positive rate of $\mathrm{HBsAg}$ and antibodies to hepatitis $\mathrm{C}$ virus (anti-HCV), serum AFP values, type of cirrhosis, tumor size, number of tumors, capsule formation, portal vein invasion, tumor differentiation, metastasis, TNM staging, resection rate, and survival rate.

During the follow-up period, patients were investigated every 2-3 months with serum AFP level, abdominal ultrasonography, abdominal computerized tomography (CT), Magnetic Resonance Imaging (MRI) or both; and celiac angiography if needed. The mean follow-up period was $24.7 \pm 20.4$ months (1-83 months).

Pathological staging of the tumor was defined by using the current UICC TNM staging system. Micro nodular cirrhosis is defined as cirrhosis with nodule less than 3 $\mathrm{mm}$ in size where as macro nodular cirrhosis is defined as cirrhosis with nodule more than $3 \mathrm{~mm}$. The data were analyzed by using SPSS statistical program. The clinical and pathological manifestations were statistically analyzed with chi-square test, Fisher's exact test or Student's t test. Survival rates were calculated by using the Kaplan-Meier method, and survival curves were compared by using the generalized Wilcoxon test. Datas were expressed as means $\pm S D$ and any $P$ value less than 0.05 was considered statistically significant.

\section{RESULTS}

During the study period (1995-1998), 1200 patients were enrolled in the study. Of these patients, 1082 patients were $\mathrm{HCC}$, and 118 patients were other pathology type. Among 1082 patients studied, 108 were elderly-HCC and 974 were younger-HCC.

\section{The survival rate and resection rate of the two groups}

The resection rate of the elderly and younger group was $91.7 \%$ (99/108), 79.7\% (776/974) respectively the resection rate of the elderly group was significantly higher than that of the younger group ( $p=0.003$ ). The mean follow-up time was $24.7 \pm 20.4$ months (183 months). The 1, 3, and 5-year survival rates were $84.6 \%, 65.3 \%$ and $59.5 \%$ respectively in the elderly group. While the 1, 3, and 5-year survival rates of the younger group were $77.8 \%, 53.4 \%$, and $45.4 \%$ respectively. The survival rates of these two groups were not statistically significant $(p=0.118)$.

\section{The clinicopathological features}

The clinicopathological features of these two groups are listed in Table 1. Compared with the younger group, the clinicopathological features of the elderly HCC patients showed the following characteristics (1) a low incidence of $\mathrm{HBsAg}$ positivity $(61.8 \%$ versus $82.1 \%$, $P=0.000),(2)$ a high incidence of anti-HCV positivity (6.4\% versus $1.7 \%, P=0.027)$, (3) a low proportion of AFP $\geq 400 \mu \mathrm{g} / \mathrm{dl}(26.9 \%$ versus $44.0 \%, P=0.001)$, (4) small diameter of the tumors $(6.20 \pm 3.74$ versus $8.13 \pm 5.14, P=0.002$ ), (5)a high proportion of stage I-II patients in TNM staging system $(64.8 \%$ versus $48.5 \%, p=0.017),(6)$ a low proportion of metastasis $(12.0 \%$ versus $20.1 \%, P=0.043)$.

There were no significant differences between two groups of patients in terms of gender, symptoms, type of cirrhosis, number of tumors, capsular formation, tumor differentiation, and portal vein invasion.

\section{DISCUSSION}

The clinical data of the patients who had undergone surgical resection for the treatment of liver cancer at Zhongshan Hospital, Fudan University, during the period of 1995-1998 were reviewed. The mean followup period was $24.7 \pm 20.4$ months ( $1-83$ months). No patient was lost during follow-up and all the data were well recorded. It should be noted that as these datas were collected from the patients who received surgical treatment from our hospital, so it can't represent the overall picture of elderly HCC patients in China.

In this study, the elderly HCC patients had a significantly low incidence of HBsAg positivity $161.8 \%$ versus $82.1 \%, P=0.000$ ). This result was similar to the results from Japan, Hongkong, and Taiwan. ${ }^{6}$ This phenomenon may have some explanations. For instance, molecular biology shows integrated HBV- DNA in both 
Jing-Lin et al. Analysis of the Clinicopathological Features of Hepatocellular Carcinoma in Elderly Patients

Table 1. Clinicopathological Features of the Elderly and Younger HCC Patients

\begin{tabular}{|c|c|c|c|c|c|}
\hline Clinicopathological features & & Elderly-HCC (\%) & Younger HCC (\%) & $\chi^{2}$ & $\mathbf{P}$ \\
\hline \multirow[t]{2}{*}{ Gender } & Male & 95 & 844 & 0.146 & 0.703 \\
\hline & Female & 13 & 130 & & \\
\hline \multirow[t]{2}{*}{ Symptoms } & Present & 62 & 635 & 2.618 & 0.106 \\
\hline & absent & 46 & 338 & & \\
\hline \multirow[t]{2}{*}{ HBsAg } & Positive & $63(61.8)$ & $742(82.1)$ & 23.66 & $0.000 *$ \\
\hline & Negative & 39 & 162 & & \\
\hline \multirow[t]{2}{*}{ Anti-HCV } & Positive & $4(6.4)$ & $9(1.7)$ & 4.861 & $0.027 *$ \\
\hline & Negative & 59 & 476 & & \\
\hline \multirow[t]{2}{*}{ AFP $(\mu \mathrm{g} / \mathrm{dl})$} & $\geq 400$ & $29(26.9)$ & $429(44.0)$ & 11.773 & $0.001 *$ \\
\hline & $\square 400$ & 79 & 545 & & \\
\hline Tumor size $(\mathrm{cm})$ & & $6.20 \pm 3.74$ & $8.13 \pm 5.14$ & l & $0.002 *$ \\
\hline \multirow[t]{3}{*}{ Cirrhotic nodule } & Absent & 14 & 111 & 1.952 & 0.377 \\
\hline & $\leq 3 \mathrm{~mm}$ & 44 & 335 & & \\
\hline & ૧3mm & 50 & 528 & & \\
\hline \multirow[t]{2}{*}{ Tumor number } & Single & 103 & 921 & 0.126 & 0.722 \\
\hline & Multiple & 5 & 53 & & \\
\hline \multirow[t]{2}{*}{ Portal vein invasion } & Present & 14 & 139 & 0.152 & 0.696 \\
\hline & Absent & 94 & 835 & & \\
\hline \multirow[t]{2}{*}{ Tumor differentiation } & $\mathrm{I}-\mathrm{II}$ & 89 & 773 & 0.556 & 0.456 \\
\hline & III-IV & 19 & 201 & & \\
\hline \multirow[t]{2}{*}{ Capsule formation } & Intact & 30 & 317 & 2.279 & 0.131 \\
\hline & Not intact & 60 & 445 & & \\
\hline \multirow[t]{3}{*}{ TNM staging } & 1 & 37 & 267 & 8.189 & $0.017 *$ \\
\hline & II & 33 & 205 & & \\
\hline & $\mathrm{III}_{\mathrm{A}}$ & 34 & 420 & & \\
\hline \multirow[t]{2}{*}{ Metastasis } & Present & $13(12.0)$ & $196(20.1)$ & 4.079 & $0.043^{*}$ \\
\hline & Absent & 95 & 778 & & \\
\hline
\end{tabular}

adult and pediatric patients. However, the integration of HBV- DNA into a single site within the host cellular DNA appears to be common in HCC tissue obtained from children, but uncommon in tumor tissues obtained from adults. ${ }^{7}$ The serum HBsAg titre may decrease with age or with superinfection of hepatitis $\mathrm{C}$ virus $(\mathrm{HCV})$ or hepatitis $D$ virus (HDV). ${ }^{8}$

The elderly HCC patients had more frequent antiHCV positivity $(6.4 \%$ versus $1.7 \%, p=0.027)$. It is reported that more HCC patients in the elderly can be attributable to chronic hepatitis or cirrhosis caused by $\mathrm{HCV}$ infection. ${ }^{9}$ Unavailability of anti HCV screening in pre world war era and large number of infected blood transfusion may be the cause of high positivity rate. The other possible explanation is most of the HBV infections in China are acquired during childhood, while HCV infections are usually acquired when one becomes adult and in those patients with history of syringe sharing or blood transfusion without screening in the past. In other words, the type of hepatitis virus infection in HCC patients is different with age group.

The elderly HCC patients had a low AFP value than that of the younger HCC patients. This result is similar to the results published by other investigators. ${ }^{10}$ The exact mechanism is unclear. The possible reason is that the tumor growth is more active in the younger patients. Further, the biological heterogeneity of cancer cells at different ages may influence the serum AFP secretion. The tendency of young patients to have larger tumors may be an alternative explanation.

The diameter of the tumor of elderly HCC patients is small compared to younger $(6.20 \pm 3.74$ versus 8.13 $\pm 5.14, P=0.002$ ). More advanced cirrhosis in elderly 
Jing-Lin et al. Analysis of the Clinicopathological Features of Hepatocellular Carcinoma in Elderly Patients

compared to young may contribute to this finding. Elderly HCC patients showed a high proportion of stage I-II in TNM staging system $164.8 \%$ versus $48.5 \%, P=$ $0.017)$, and a low proportion of metastasis $(12.0 \%$ versus $20.1 \%, P=0.043$ ). The possible reasons might be that the surgeons become more prudent in making a decision of resection in the elderly patients. The degree of malignancy in elderly is lower than younger patients and the rate of growth is slower than younger patients. That's the similar reason, which account for the higher resection rate of tumor in elderly patients $191.7 \%$ versus $79.7 \%$ ).

The 1, 3 and 5-year survival rate of the elderly group was slightly higher than that of the younger group, but there was no statistically significant difference between these two groups $(P=0.018)$. If the indication of resection is strictly followed, the post-operative survival rate of the elderly patients is as comparable as that of the younger patients of HCC. ${ }^{11}$

\section{CONCLUSIONS}

This study shows that the difference in clinicopathological features really exists between the elderly and younger HCC patients. There are age-related differences in the clinicopathological features of HCC patients. Different mechanisms of hepatocarcinogenesis may exist between younger and elderly HCC patient, which in turn makes the clinicopathological differences between the two groups. So, for the management of HCC patients older than 65 years, if there is no contraindication for surgery, resection still is the first choice of treatment.

\section{REFERENCES}

1. Yang BH, Xia JL, Huang LW, et al. Changed clinical aspects of primary liver cancer in China during the past 30 years. Hepatobiliary Pancreat Dis Int 2004; 3 (2):194-198

2. Health Statistics of the Republic of China. Vital Statistics, Taipei, Taiwan, R.O.C., 1992

3. Sandler DP, Sandler RS, Horney LF. Primary liver cancer mortality in the United States. J Chron Dis 1983; 36:227-36.

4. Dohmen K, Shirahama M, Shigematsu H, Irie K, Ishibashi H. Optimal treatment strategy for elderly patients with hepatocellular carcinoma. J Gastroenterol Hepatol 2004;19(8):859-865.

5. Tsutomu N, Akira K, Naoki S, Yukifumi K, Junichi U. Agerelated, different clinicopathological features of HCC patients Ann Surg 1995; 221:308-14

6. Yeh CN, Lee WC, Jeng LB, Chen MF. Hepatic resection for hepatocellular carcinoma in elderly patients. Hepatogastroenterology 2004; 51(55): 219-23.
7. Chang MH, Chen PJ, Chen JY, Lai MY, Hsu HC, Lain DC et al. Hepatitis B virus integration in hepatitis B-related hepatocellular carcinoma in childhood. Hepatology 1991; 13:316-20.

8. Wu JC, Chen TZ, Huang YS, Yen FS, Ting LT, Sheng WY et al. Natural history of hepatitis D viral superinfection significance of viremia detected by polymerase chain reaction. Gastroenterology 1995; 108:796-802.

9. Nishioka K, Watanabe J, Furuta S, Tanaka E, Iino S, Suzuki H et al. A high prevalence of antibody to the hepatitis $C$ virus in patients with hepatocellular carcinoma in Japan. Cancer 1991; 67:429-33.

10. Kew MC, Maccrollo P. Effect of age on the etiologic role of the hepatitis B virus in hepatocellular carcinoma in blacks. Gastroenterology 1988; 94:439-42.

11. Nagasue N, Chang YC, Takemoto $Y$, Taniura H, Kohno H, NakamuraT. Liver resection in theaged (seventy years or older) with hepatocellular carcinoma. Surgery 1993; 113:148-54. 